Georgian Mathematical Journal

Volume 11 (2004), Number 4, 603-611

\title{
COMPLEX POINTS OF TWO-DIMENSIONAL SURFACES
}

\author{
T. ALIASHVILI
}

Dedicated to the memory of Academician G. Chogoshvili

\begin{abstract}
We deal with complex points of two-dimensional surfaces. A short review of basic results about complex points of smooth surfaces in $\mathbb{C}^{2}$ is presented at the beginning. For algebraic surfaces, a formula is proved which expresses the number of complex points as the local degree of an explicitly constructible polynomial endomorphism.
\end{abstract}

2000 Mathematics Subject Classification: 32S05, 55M25.

Key words and phrases: Surface, grassmannian, complex point, Euler characteristic, mapping degree.

\section{INTRODUCTION}

Complex points of smooth two-dimensional surfaces in $\mathbb{C}^{2}$ play a significant role in some problems of complex analysis [5], [9]. For example, the existence of complex points sometimes enables one to construct the so-called attached analytic discs with boundaries in a given surface [5], [13]. Correspondingly, complex points are related to the so-called Bishop problem about the existence of analytic discs attached to the graph of an arbitrary smooth complex-valued function in the plane [5]. Despite considerable progress, this problem has not yet been completely solved and complex points of two-dimensional surfaces attract permanent interest [13]. Moreover, complex points of surface germs appear in some issues of singularity theory [4], [13].

There are also some open problems concerned with complex points on compact surfaces [9]. It is remarkable that in the setting of compact surfaces this problem has interesting topological aspects. In particular, it is well-known that the geometry of complex points on a compact surface $X$ is closely related to its Euler characteristic $\chi(X)[7],[9]$.

Recently, it has turned out that some of the above topics can be successfully treated in the case where a surface is given by polynomial equations, using the so-called signature formulae for topological invariants [12]. For example, this approach is quite effective in the case of the graph of a polynomial planar endomorphism [13]. One can also consider a complete intersection defined by two polynomials on $\mathbb{C}^{2} \cong \mathbb{R}^{4}$. In such situations it is possible to find the number of complex points in terms of local degrees of explicitly constructible polynomial endomorphisms. The aim of this paper is to present a new result of such kind. 
We start by recalling some relevant definitions and techniques. The main formula for the number of complex points on an algebraic surface in $\mathbb{C}^{2}$ is presented in the second section (Theorem 2.1). We also obtain an upper estimate for the number of complex points on an algebraic surface (Theorem 2.2).

\section{Algebraic Formulae for Topological Invariants}

The main goal of this paper being the obtaining of an explicit formula for the number of complex points, we begin by recalling some results about the counting of real roots of polynomial equations. We basically follow [12] with slight modifications made to fit into the context of the present paper.

Before giving the precise formulation of the result we need to introduce some notation. Let $f: U \rightarrow \mathbb{R}^{n}$ be a smooth mapping defined in the neighbourhood $U$ of the origin 0 in $\mathbb{R}^{n}$ such that $f(0)=0$. In this case we say that the origin is a zero of $f$. Equivalently, one can deal with a smooth map-germ $f:\left(\mathbb{R}^{n}, 0\right) \rightarrow\left(\mathbb{R}^{n}, 0\right)$ as in $[8]$.

Let $\mathbb{R}[n]$ be the algebra of the formal power series in $n$ indeterminates with real coefficients. Denote by $A$ the local algebra of $f$ at the origin, i.e., $A=$ $\mathbb{R}[n] /(\tilde{f})$, where $(\tilde{f})$ is the ideal generated by the formal Taylor expansions of functions $f_{j}$ at the origin.

The fundamental assumption in this section is that $A$ is finite-dimensional, in which case we say that $f$ is of finite multiplicity at the origin (in the language of germs one just says that $f$ is a finite map germ [8]). Thus we assume that $A$ is a finite-dimensional algebra over $\mathbb{R}$. Denote by $j$ the class of Jacobian of $f$ in $A$.

It is well known that the principal ideal $(j)$ generated by $j$ in $A$ consists of real multiples of $(j)$, i.e., $(j)=\mathbb{R} j$. This implies that for every $a \in(j)$ there exists a unique $r \in \mathbb{R}$ such that $a=r j$. In this case we can write $r=\frac{a}{j}$ and speak of its sign $\operatorname{sgn} r$ if $r \neq 0$.

For an ideal $I$ of $A$, let $I^{2}$ denote its square (the ideal generated by all pairwise products of the elements from $I)$. Denote by $N(A)$ the set of all ideals in $A$ having zero squares, i.e.,

$$
N(A)=\left\{I \text { is an ideal in } A: I^{2}=0\right\} .
$$

For any subset $X \subset A$, put ann $X=\{a \in A: a X=0\}$. Obviously, for $I \in N(A), I \subset$ ann $I$. Finally, let us notice that under the assumption of finite multiplicity the origin is an isolated zero of $f$, in other words, 0 is isolated in $f^{-1}(0)$. As it is well known, in this case one can define the local topological (mapping) degree $\operatorname{deg}_{0} f[12]$.

Theorem 1.1 ([8], [11]). Let $f$ be a smooth mapping of finite multiplicity at the origin. Then, for an arbitrary maximal element $N$ of $N(A)$, the inclusion $(\operatorname{ann} N)^{2} \subset(j)$ takes place. Moreover, if ann $N=N$ then $\operatorname{deg}_{0} f=0$, and if $N \subset$ ann $N$ then, for an arbitrary element $a \in$ ann $N-N$, one has

$$
\operatorname{deg}_{0} f=\operatorname{sgn}\left(\frac{a^{2}}{j}\right)\left(\operatorname{dim}_{\mathbb{R}} \operatorname{ann} N-\operatorname{dim}_{\mathbb{R}} N\right) .
$$


This formula plays an important role in many problems concerning computation of various invariants of the polynomial mappings and real algebraic sets. We emphasize that all algebraic constructions used in this formula can be effectively performed in each concrete case, so the local degree is algorithmically computable.

As shown in [6], [12], the Euler characteristic of a compact algebraic set can be expressed through the local degree of an auxiliary polynomial mapping. We reproduce the corresponding general formula from [6]. Let $X$ be an affine algebraic subset of $\mathbb{R}^{n}$. Obviously, every such subset may be represented as a hypersurface $X=\{F=0\}$ with $F=f_{1}^{2}+\cdots+f_{k}^{2}$, where $f_{j}$ are the polynomials defining $X$. For a finite $X$, the Euler characteristic simply reduces to the number of geometrically distinct points.

We work with the usual notion of the Euler characteristic $\chi(X)$ defined as the alternating sum of the ranks of homology groups of a topological space $X$ [7]. We only consider the homology with integer coefficients [7]. As always, we write $\operatorname{deg}_{p} f$ for the local degree of an endomorphism $f: \mathbb{R}^{n} \rightarrow \mathbb{R}^{n}$ for any $p \in f^{-1}(0)$ which is an isolated pre-image of the origin. Recall that $\operatorname{deg}_{p} f$ is defined as the topological degree of the mapping

$$
\widehat{f}=f /\|f\|: S_{\varepsilon}^{n-1}(p) \rightarrow S_{1}^{n-1}(0)=S^{n-1},
$$

where $\varepsilon$ is a sufficiently small positive number [12].

Theorem $1.2([6],[12])$. Let $f_{1}, \ldots, f_{p} \in \mathbb{R}_{n}$ be real polynomials of degrees not exceeding d. Suppose that the set $X$ of their common real zeroes is compact. Set

$$
\begin{gathered}
h_{i}\left(x_{0}, x_{1}, \ldots, x_{n}\right)=x_{0}^{d+1} f_{i}\left(\frac{x_{1}}{x_{0}}, \ldots, \frac{x_{n}}{x_{0}}\right), \\
H=\sum_{i=1}^{p} h_{i}^{2}-\sum_{k=0}^{n} x_{k}^{2 d+4} .
\end{gathered}
$$

Then $H$ has an algebraically isolated critical point at the origin $\mathbb{O} \in \mathbb{R}^{n+1}$ and the following equality holds:

$$
2 \chi(X)=(-1)^{n}-\operatorname{deg}_{\mathbb{O}}(\nabla H),
$$

where $\nabla H$ denotes the gradient of the polynomial $H$.

If the set $X$ is finite this formula gives the number of points in $X$, i.e. the number of real solutions of the corresponding polynomial system. Notice that here the solutions are counted without taking into account their multiplicities. From the algebraic formula for local degree it follows that the Euler characteristic on the left-hand side of (1.2) is effectively computable in each concrete case. This theorem enables us to give an explicit formula for the number of complex points of an algebraic smooth surface in $\mathbb{C}^{2}$.

We also recall a general estimate for the topological degree in terms of the socalled Petrovsky numbers which were introduced in [3], [14]. The term "integer point" means a point of the integer lattice $\mathbb{Z}^{n} \subset \mathbb{R}^{n}$. 
Definition 1.1. For an $n$-tuple of natural numbers $\mathbf{m}=\left(m_{1}, \ldots, m_{n}\right)$, define $P_{n}(\mathbf{m})$ as the number of all integer $n$-tuples $k=\left(k_{1}, \ldots, k_{n}\right)$ satisfying conditions:

$$
0 \leq k_{i} \leq m_{i}-1, \quad i=1, \ldots, n ; \quad \sum_{i=1}^{n} k_{i}=\sum_{i=1}^{n} \frac{m_{i}-1}{2} .
$$

By analogy with [3], the number $P_{n}(\mathbf{m})$ may be called a $P$-number of type $(n, \mathbf{m})$. If all numbers $m_{j}$ are equal, say to $m$, we write $P_{n}(m)$ instead of $P_{n}(m, \ldots, m)$.

Theorem 1.3 ([14]). Let $F=\left(f_{1}, \ldots, f_{n}\right)$ be a homogeneous endomorphism of $\mathbb{R}^{n}$ of multi-degree $\mathbf{m}$. Then

$$
|\operatorname{deg} F| \leq P_{n}(\mathbf{m}) .
$$

A. Khovansky established the exactness of the above estimate and applied it to some problems of real algebraic geometry [14]. We use this to estimate a maximal possible number of complex points on an algebraic surface.

\section{Counting Complex Points}

We need first some notions and constructions concerned with the grassmannian $G=G r_{+}(2,4)$ of oriented two-planes in $\mathbb{R}^{4}=\mathbb{C}^{2}$. As is well known [7], $G$ is a compact four-dimensional smooth manifold homeomorphic to $S^{2} \times S^{2}$. Actually, this manifold can be embedded in $\mathbb{R P}^{5}$ using the so-called Plücker embedding which gives rise to natural coordinates on $G[7]$. We will use a version of this construction to introduce coordinates on $G$ appropriate for our purposes.

For us it is important to distinguish between complex lines (i.e., one-dimensional complex subspaces of $\mathbb{C}^{2}$ ) and the so-called totally real planes in $\mathbb{C}^{2}$, so we recall that a plane $V \in G$ is called totally real if $V+\imath V=\mathbb{C}^{2}$. We denote by $G_{\mathbb{R}}$ the subset of $G$ consisting of totally real planes.

Another natural subset is the collection of all complex lines endowed with their natural (intrinsic complex) orientations defined by multiplication by $\imath$. It is clearly homeomorphic to the Riemann sphere $\mathbb{C P}^{1}$ and will be denoted by $\mathbb{C P}_{+}$. Another copy $\mathbb{C P}_{-}$of the Riemann sphere is obtained by taking all complex lines with the opposite orientations. Thus the collection $G_{\mathbb{C}}$ of all oriented complex lines is homeomorphic to the union of two disjoint copies of $S^{2}$. These and all other submanifolds of $G$ will always be endowed with natural orientations coming from the complex structure in $\mathbb{C}^{2}$.

Let $X$ be a smooth two-dimensional surface in $\mathbb{C}^{2}$. Some important features of its position in $\mathbb{C}^{2}$ are characterized by local behaviour at points where the tangent plane is a complex line [5]. In the setting used in this paper, $X$ is given by two algebraic equations in $\mathbb{C}^{2}$, so it can be oriented in the usual way using the gradients of defining functions. Then each tangent plane acquires an orientation induced from $X$ and can be considered as an element of $G$. If a tangent plane appears to be a complex line, we can compare its orientation induced from $X$ with its natural orientation, so that it can be attributed either 
to $\mathbb{C P}_{+}$or to $\mathbb{C P}_{-}$. The same is applicable for any smooth oriented surface in $\mathbb{C}^{2}$.

Definition 2.1. Let $X$ be a smooth oriented two-dimensional surface in $\mathbb{C}^{2}$. A point $p \in X$ is called a complex point of $X$ if the tangent plane $T_{p} X$ is a complex line in $\mathbb{C}^{2}$. A complex point is said to be positive if $T_{p} X \in \mathbb{C P}_{+}$and negative in the opposite case.

The set of all complex points of $X$ is denoted by $\mathbb{C}(X)$. If it is finite its cardinality is denoted by $c(X)$. It is known that, for a generic surface $X$, the set of complex points is finite [9]. Our main concern is to establish effective criteria for complex points and methods of computing $c(X)$. In the sequel we need the following interpretation of complex points.

Consider the Gauss mapping $\Gamma: X \rightarrow G$. Then its image is a two-dimensional compact surface in the four-dimensional manifold $G$. Obviously, a point $p$ is a complex point iff $\Gamma(x) \in G_{\mathbb{C}}$. Thus the complex points correspond to the intersections of $\Gamma(X)$ with $G_{\mathbb{C}}$. Notice that the latter manifold is also twodimensional, so one can define the local intersection number of these two submanifolds in $G$ [7]. Let us call it the index of a complex point $p$.

Generically, these two submanifolds intersect transversally in which case we say that a complex point is transversal. It is easy to verify that this is equivalent to the fact that the local intersection index at this point is equal to \pm 1 and this complex point is preserved under small perturbations of $X$. For this reason, transversal complex points are sometimes called generic [13].

In the general case, where complex points are isolated but not necessarily transversal, one can consider the intersection number of $\Gamma(X)$ and $G_{\mathbb{C}}$ in $G$ (this is possible because all these manifolds are oriented). It is called the total index of $X$. As usual, the intersection number can be computed as the sum of local intersection indices. For a generic $X$, all complex points are transversal so in this case the total index is just the algebraic number of complex points [12].

We are basically interested in the case where $X$ is an algebraic surface given by explicit equations. Denote by $\mathbb{R}_{n}$ the algebra of real polynomials in $n$ variables. Let $f, g \in \mathbb{R}_{4}$ be two polynomials in four indeterminates and $X=\{f=0, g=0\}$ be the set of their common zeroes in $\mathbb{R}^{4} \cong \mathbb{C}^{2}$. We aim now at finding effective methods of computing $c(X)$. As a first step we obtain explicit equations defining complex points of $X$ in $\mathbb{C}^{2}$ in terms of the coordinates on $G$.

Recall that the coordinates on $G$ can be introduced as follows (this construction goes back to Grassmann himself [7]). Let $P \in G$ be a two-plane. Choose a basis $\left(v_{1}, v_{2}\right)$ in $P$. Taking the coordinates of these two vectors as rows one obtains a $(2 \times 4)$-matrix. Now the standard coordinates of $P$ are defined as six $(2 \times 2)$-minors of this matrix. They can be denoted by $m_{i j}$, where $i, j$ are the numbers of columns contained in a given minor. It is convenient to renumber them in "antilexicographic" order by putting

$$
s_{1}=m_{12}, \quad s_{2}=m_{13}, \quad s_{3}=m_{14}, \quad s_{4}=m_{23}, \quad s_{5}=m_{24}, \quad s_{6}=m_{34} .
$$


The numbers $s_{j}$ of course depend on a choice of the basis $\left(v_{1}, v_{2}\right)$ and so they should be considered as projective coordinates [7]. As is well known, they satisfy the quadratic relation

$$
s_{1} s_{6}-s_{2} s_{5}+s_{3} s_{4}=0,
$$

which shows that they define a mapping of $G$ into a quadric defined by this equation in $\mathbb{R P}^{5}$. This mapping is actually an embedding and so in such a way one gets an atlas of local coordinates on $G$ which we also call standard coordinates. Actually, the equations which we are going to derive are homogeneous, so for our purposes it is possible to work directly with the numbers $s_{j}$. The next step is to write conditions on the coordinates $s_{j}$ which distinguish complex lines.

To this end, consider a complex line $P \in G$. Since $P$ is invariant under multiplication by $\imath$, it admits a basis of the form $(v, v v)$, where $v$ is any non-zero vector in $P$. Denoting by $\left(a_{1}, a_{2}, a_{3}, a_{4}\right)$ the coordinates of $v$ we see that the second row of the associated $(2 \times 4)$-matrix is then $\left(-a_{2}, a_{1},-a_{4}, a_{3}\right)$. Computing the minors $m_{i j}$, one notices that, in addition to the quadratic relation $(2.1)$, in this case one has two linear relations:

$$
s_{2}=s_{5}, \quad s_{3}=-s_{4}
$$

In view of the linearity, the validity of these relations does not depend on a choice of the basis in $P$. It is also easy to see that these relations are independent (as functions of the coordinates $s_{j}$ ) and that each 2-plane in $\mathbb{C}^{2}$ with standard coordinates satisfying these relations is a complex line. Thus (2.2) are exactly the defining equations of $G_{\mathbb{C}}$ in $G$.

Returning to the situation of a two-surface $X$ notice that, at each point $p \in X$, the gradients $\nabla_{p} f, \nabla_{p} g$ are orthogonal to the tangent plane $T_{p} X$ (with respect to the standard scalar product in $\mathbb{R}^{4} \cong \mathbb{C}^{2}$ ). It is well known and easy to see that a 2-plane is a complex line if and only if its orthogonal complement is a complex line. Thus we see that $T_{p} X$ being a complex line is equivalent to the validity of equations $(2.2)$ for the $(2 \times 4)$-matrix formed by $\nabla_{p} f, \nabla_{p} g$. In other words, $p \in X$ is a complex point of $X$ if and only if

$$
f_{1} g_{3}-f_{3} g_{1}-f_{2} g_{4}+f_{4} g_{2}=0, \quad f_{1} g_{4}-f_{4} g_{1}+f_{2} g_{3}-f_{3} g_{2}=0,
$$

where the subscripts denote partial derivatives with respect to the corresponding variable taken at the point $p$. By adding the equations of $X$ we get a $(4 \times 4)$ system of real polynomial equations, the real roots of which give complex points of $X$ in $\mathbb{C}^{2}$.

Thus for finding the number of complex points one can directly apply Theorem 1.2. To make this completely explicit let us write the auxiliary functions appearing in the latter theorem. Suppose, for simplicity, that $f, g \in \mathbb{R}_{4}$ have the same algebraic degree $m \geq 2$. Then obviously all their partial derivatives have algebraic degrees not exceeding $m-1$, so both functions appearing in equations (2.3) have algebraic degrees not exceeding $2(m-1) \geq m$. Thus for applying Theorem 1.2 we should take $d=2 m-2$ and $n=4$. 
Then following Theorem 1.2 we introduce the polynomials:

$$
\begin{gathered}
h_{1}(t, x, y, u, v)=t^{d+1} f\left(\frac{x}{t}, \frac{y}{t}, \frac{u}{t}, \frac{v}{t}\right), h_{2}(t, x, y, u, v)=t^{d+1} g\left(\frac{x}{t}, \frac{y}{t}, \frac{u}{t}, \frac{v}{t}\right), \\
h_{3}(t, x, y, u, v)=t^{d+1}\left[\left(\frac{\partial f}{\partial x} \frac{\partial g}{\partial u}-\frac{\partial f}{\partial u} \frac{\partial g}{\partial x}-\frac{\partial f}{\partial y} \frac{\partial g}{\partial v}+\frac{\partial f}{\partial v} \frac{\partial g}{\partial y}\right)\left(\frac{x}{t}, \frac{y}{t}, \frac{u}{t}, \frac{v}{t}\right)\right], \\
h_{4}(t, x, y, u, v)=t^{d+1}\left[\left(\frac{\partial f}{\partial x} \frac{\partial g}{\partial v}-\frac{\partial f}{\partial v} \frac{\partial g}{\partial x}+\frac{\partial f}{\partial y} \frac{\partial g}{\partial u}-\frac{\partial f}{\partial u} \frac{\partial g}{\partial y}\right)\left(\frac{x}{t}, \frac{y}{t}, \frac{u}{t}, \frac{v}{t}\right)\right], \\
H=h_{1}^{2}+h_{2}^{2}+h_{3}^{2}+h_{4}^{2}-\left(t^{2 d+4}+x^{2 d+4}+y^{2 d+4}+u^{2 d+4}+v^{2 d+4}\right),
\end{gathered}
$$

where $d=2 m-2$ and $(x, y, u, v)$ denote canonical real coordinates in $\mathbb{C}^{2}$. Now from Theorem 1.2 we get an explicit formula for the number of complex points.

Theorem 2.1. For a generic $X \subset \mathbb{C}^{2}$, the number of complex points of $X=\{f=0, g=0\} \subset \mathbb{C}^{2}$ is given by

$$
c(X)=\frac{1}{2}\left(1-\operatorname{deg}_{\mathbb{O}} \nabla H\right),
$$

where $H$ is given by (2.4).

We can now obtain a general estimate for the number of complex points on an algebraic surface defined by equations of fixed algebraic degrees. Below, by saying "generic" we mean that all complex points are isolated.

Theorem 2.2. The number of complex points of a generic algebraic surface defined by two equations of degree $m \geq 2$ does not exceed $P_{5}(4 m-1)$.

This follows from the proof of Theorem 2.1. Indeed, we have seen that in our case the algebraic degrees of polynomials $h_{j}$ do not exceed $2 m-2$. So the algebraic degree of $H$ does not exceed $4 m$ and that of its gradient does not exceed $4 m-1$. Taking into account that $n=4$ we get the desired inequality directly from Theorem 1.3.

This result can be considered as an upper estimate for the number of complex points. Notice that it also implies that if complex points are isolated then their number is finite even if the surface is not compact. For compact surfaces, it would be interesting to find an exact range of values of $c(X)$ if the degrees of defining equations are fixed. It is also natural to ask what a range of $c(X)$ is for generic compact surfaces of fixed genus $g$.

In relation with the latter problem we add that a lower estimate for the number of complex points can be given in topological terms. For a generic algebraic surface $X \subset \mathbb{C}^{2}$, denote by $C(X)$ the number of complex points counted with multiplicities, i.e. the sum of absolute values of the local intersection indices over all complex points. If all complex points are transversal one has $C(X)=c(X)$.

Theorem 2.3. For a generic compact surface $X$, one has

$$
C(x) \geq|\chi(X)| \text {. }
$$

This result is known in the case where all complex points are transversal [9]. The general case follows by the continuity argument. 
In general, the number of complex points may change under deformations of $X$ but there is another related characteristic which is topologically invariant. Denote by $\mathbb{C P}_{+} \cup-\mathbb{C P}_{-}$the union of these two submanifolds of $G$ where the first one is taken with its natural orientation and the second one is taken with the reversed orientation.

Definition 2.2. The Maslov index of $X$ is defined as the intersection index of $\Gamma(X)$ with $\mathbb{C P}+\cup-\mathbb{C P}_{-}$.

From the results of [12] it follows that the intersection index of two explicitly given submanifolds can be effectively calculated as the local degree of an auxiliary polynomial endomorphism. Using the standard coordinates on $G$ it is not difficult to write explicit equations for $\Gamma(X)$ while equations for $\mathbb{C P}_{+} \cup-\mathbb{C P}_{-}$ are provided by (2.2). We do not write the resulting formula in terms of the local degree since it is rather complicated but formulate the main conclusion.

Theorem 2.4. The Maslov index of X can be algorithmically computed in terms of the coefficients of $f$ and $g$.

As is well known, the total index and the Maslov index are the main numerical invariants characterizing the complex geometry of $X$. There are many results about possible values of these invariants and their behaviour under various transformations and constructions [9]. Using our Theorem 2.1 and the computer program for calculating the topological degree [15] one can easily compute the number of complex points in concrete examples.

\section{REFERENCES}

1. T. Aliashvili, Counting real roots of polynomial endomorphisms. J. Math. Sci. 118(2003), No. 5, 5325-5346.

2. T. Aliashvili, On invariants of random planar endomorphisms. Banach Center Publ. 62(2004), 19-28.

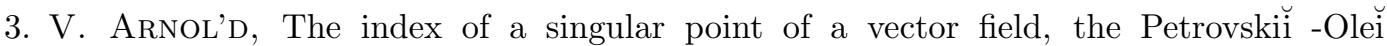
nik inequalities, and mixed Hodge structures. (Russian) Funkcional. Anal. i Prilozhen. 12(1978), No. 1, 1-14; English transl.: Funct. Anal. Appl. 12(1978), 1-12.

4. V. I. Arnol'D, A. N. Varchenko, and S. M. Gusě̌n-Zade, Singularities of differentiable mappings. (Russian) Nauka, Moscow, 1982.

5. E. Bishop, Differentiable manifolds in complex Euclidean space. Duke Math. J. 32(1965), $1-21$.

6. J. W. Bruce, Euler characteristics of real varieties. Bull. London Math. Soc. 22(1990), No. 6, 547-552.

7. B. A. Dubrovin, S. P. Novikov, and A. T. Fomenko, Modern geometry. (Russian) Nauka, Moscow, 1979.

8. D. Eisenbud and H. I. Levine, An algebraic formula for the degree of a $C^{\infty}$ map germ. With an appendix by Bernard Teissier, "Sur une inégalité à la Minkowski pour les multiplicités". Ann. Math. (2) 106(1977), No. 1, 19-44.

9. F. Forstnerič, Complex tangents of real surfaces in complex surfaces. Duke Math. J. 67(1992), No. 2, 353-376. 
10. G. Ishikawa and T. Онмото, Local invariants of singular surfaces in an almost complex four-manifold. Ann. Global Anal. Geom. 11(1993), No. 2, 125-133.

11. G. Khimshiashvili, The local degree of a smooth mapping. (Russian) Soobshch. Akad. Nauk Grusin. SSR 85(1977), No. 2, 309-312.

12. G. Khimshiashvili, Signature formulae for topological invariants. Proc. A. Razmadze Math. Inst. 125(2001), 1-121.

13. G. Khimshiashvili and E. Wegert, Analytic discs and complex points of surfaces. Bull. Soc. Sci. Lett. Lódz Sér. Rech. Déform. 38(2002), 147-166.

14. A. Khovanskil, The index of a polynomial vector field. (Russian) Funktsional. Anal. $i$ Prilozhen. 13(1979), No. 1, 49-58.

15. A. Lecki and Z. Szafraniec, An algebraic method for calculating the topological degree. Topology in nonlinear analysis (Warsaw, 1994), 73-83, Banach Center Publ., 35, Polish Acad. Sci., Warsaw, 1996.

(Received 26.03.2004)

Author's address:

Teimuraz Aliashvili

Department of Mathematics No. 99

Georgian Technical University

77, Kostava St., Tbilisi 0175

Georgia

E-mail: aliashvili@mail.ge 